\title{
ASO-TDMA: ad-hoc self-organizing TDMA protocol for shipborne ad-hoc networks
}

\author{
Changho Yun ${ }^{*}$ and Yong-kon Lim
}

\begin{abstract}
A shipborne ad-hoc network (SANET), a maritime counterpart of the terrestrial vehicle ad-hoc network, can provide ships with diverse multimedia services by substituting digital maritime VHF communications for expensive satellite communications. This article proposes ad-hoc self-organizing TDMA (ASO-TDMA), a medium access control (MAC) protocol targeting SANETs. Frames in ASO-TDMA are divided into several sub-frames, and based on the proposed rules for assigning time slots, ships can only reserve time slots for data transfers through their available sub-frames. Accordingly, ASO-TDMA provides better performance in terms of reducing receiver collisions from hidden terminal problems compared to self-organizing TDMA (SO-TDMA) and carrier-sensing TDMA (CS-TDMA), two existing MAC protocols for maritime VHF communications. In addition, the article compares the performance of the three MAC protocols in terms of delays and collision rates (CRs). The results suggest that, given the same delay, ASO-TDMA can reduce the CR by as much as 30\% in comparison with SO-TDMA and CS-TDMA. Similarly, given the same CR, ASO-TDMA can reduce delays by as much as $26 \%$ in comparison with SO-TDMA and CS-TDMA.
\end{abstract}

Keywords: Ad-hoc, Communication, MAC, Maritime, Network, VHF

\section{Introduction}

Maritime communication systems such as the global maritime distress and safety system [1] and the automatic identification system (AIS) [2] have focused mainly on ship security, location tracking, identification, and surveillance. Although small-sized text message transfers based on narrow-band direct printing [3] or navigational telex [4] have been introduced, maritime communication systems have generally lacked versatility in comparison with terrestrial wireless communication systems because it has been considered premature for ships to provide multimedia services.

Recently, there has been a gradual increase in ship's demand for various multimedia services such as fullduplex digital data transfers, video streaming, and even internet access. Without loss of generality, satellite communication systems may be a good candidate for realizing such multimedia services because they can reliably support high data rates as well as offer immense communication coverage. However, satellite communications can be

*Correspondence: sgn0178@gmail.com

Ocean System Engineering Research Division, KIOST, Yuseong-daero, Yuseong-gu, Daejeon 305-343, Korea burdensome for ship subscribers because of its remarkably high cost. At sea, where a ship rarely finds other ships referred to as neighboring ships (NSs), satellite communications may be an inevitable option for obtaining multimedia services. On the other hand, a ship can easily locate NSs near the shore. In this case, we can take advantage of ad-hoc communication between ships by using another radio frequency band such as the terrestrial mobile ad-hoc network or the vehicle ad-hoc network. We refer to a new maritime ad-hoc network replacing satellite communications as a shipborne ad-hoc network (SANET), as defined in [5]. Hence, a SANET near the shore, together with satellite communications at sea, may offer consistent and affordable multimedia services.

Based on current standardization activities through the World Radiocommunication Conference (WRC) [6], maritime digital communications in VHF bands are considered a good counterpart of satellite communications for multimedia services. VHF communications can support relatively high data rates (tens of kbps). These data rates can increase to hundreds of kbps through orthogonal frequency division multiplexing (OFDM) [7,8]. Accordingly, the physical layer specifications recommended for the maritime digital VHF communication systems in [7],

\section{Springer}

(c) 2012 Yun and Lim; licensee Springer. This is an Open Access article distributed under the terms of the Creative Commons Attribution License (http://creativecommons.org/licenses/by/2.0), which permits unrestricted use, distribution, and reproduction in any medium, provided the original work is properly cited. 
including those for multiplexing, modulation, and channel coding, can be applicable to SANETs.

A SANET must efficiently manage channel access for randomly deployed ships at sea by considering the inherent infrastructure-less network environment. Thus, SANETs demand a decentralized and predictable medium access control (MAC) protocol in the data link layer. There are two MAC candidates for SANETs. One is IEEE 802.11 carrier sense multiple access/collision avoidance (CSMA/CA), which is a contention-based MAC protocol used for terrestrial ad-hoc networks [9]. The other is a contention-free time division multiple access (TDMA) based MAC protocol in which the ships' channel access is not centrally controlled but decentralized under the given frame. The latter includes self-organizing TDMA (SO-TDMA) and carrier-sensing TDMA (CS-TDMA). These two MAC candidates are similar in that ships sense the channel status. When a channel is busy, CSMA/CA goes to a random back-off, but SO-TDMA or CS-TDMA searches for other idle time slots in the current frame. Although CSMA/CA is currently employed in terrestrial ad-hoc networks, it has not been verified as a MAC protocol for a maritime counterpart under any standards. On the other hand, SO-TDMA and CS-TDMA are recommended as MAC protocols for maritime VHF communications and are currently used for the AIS, one of the most widely used maritime services [2,7]. In addition, CSMA/CA entails unbounded delays in heavy traffic environments and receiver collisions from hidden terminal problems. Accordingly, we focus only on contention-free TDMA-based MAC protocols for SANETs.

SO-TDMA and CS-TDMA cannot directly be applicable to SANETs because they are only designed for one-hop communication between a base station and a ship. Thus, there is a need for an appropriate MAC protocol for SANETs that address the following aspects:

- Ad-hoc manner: although both SO-TDMA and CSTDMA are suitable for peer-to-peer communications regarding ship communications or even vehicular communications, they do not consider ad-hoc communication. Since SANETs are based on ad-hoc communication, a ship can be a relay node between a source and a destination. Data from a source may disappear while being forwarded by several relay ships [10]. Thus, a MAC protocol for SANETs needs to consider reliable data transfers (rdts) and guarantee complete end-to-end data transfers without failure.

- Frame length: in SO-TDMA or CS-TDMA, ships just send information on their identity (e.g., vessel numbers, locations, and IMO numbers) within a single frame lasting $1 \mathrm{~min}$ [2]. In a SANET, it is necessary to consider other multimedia services such as data transfers, e-mails, text messages, and video streaming. Accordingly, a MAC protocol for SANETs should be designated to reduce the overall delay by reducing the length of a frame, to a greater extent than SO-TDMA and CS-TDMA.

- Receiver collision: SO-TDMA and CS-TDMA experience receiver collisions from hidden terminal problems. For example, two ships that cannot sense each other reserve the same time slot and send data to the same NS [11]. Thus, a MAC protocol for SANETs must mitigate this problem of receiver collisions.

Given the above aspects, we propose ad-hoc selforganizing TDMA (ASO-TDMA), a MAC protocol for SANETs that can reduce the length of a frame for multimedia services requiring high data rates. ASO-TDMA can manage SANETs' ad-hoc environments by allowing for rdts, one-hop data, non-acknowledgement (NACK), timeout, and retransmission. In addition, ASO-TDMA can reduce the possibility of receiver collisions. For this, each frame is fragmented into several sub-frames, and a network is divided into several hops. A hop is defined as a zone determined by its distance from a base station. A ship located in an arbitrary hop can only reserve time slots in available sub-frames based on the proposed rules for the assignment of time slots, which helps ships to avoid receiver collisions. Thus, ASO-TDMA is expected to outperform SO-TDMA and CS-TDMA in terms of receiver collisions and overall delays.

The rest of this article is organized as follows: "SANET architecture" section discusses the SANET architecture, "Previous works" section provides a review of previous research on SO-TDMA and CS-TDMA. "ASO-TDMA for SANETs" section presents ASO-TDMA by phase. "Performance analysis" section analyzes the performance of ASO-TDMA in terms of delays and collision rates (CRs) and compares it with that of SO-TDMA and CS-TDMA, and the article ends with "Conclusions" section.

\section{SANET architecture}

Figure 1 illustrates the SANET architecture consisting of several ships and a base station. The base station can provide ships with various maritime multimedia services by operating application servers. In addition, the base station, which is connected to IP-based backbone networks, enables the ships to support IP-based multimedia services. The ships, which are equipped with maritime VHF communication systems, can use these multimedia services by connecting to the base station when they are near the shore. In addition, the ships can send data to other ships and can communicate with one another by using the VHF communication systems.

The topology of a SANET is not fixed because all ships at sea navigate toward their destinations with different directions and speeds although several ships may navigate 


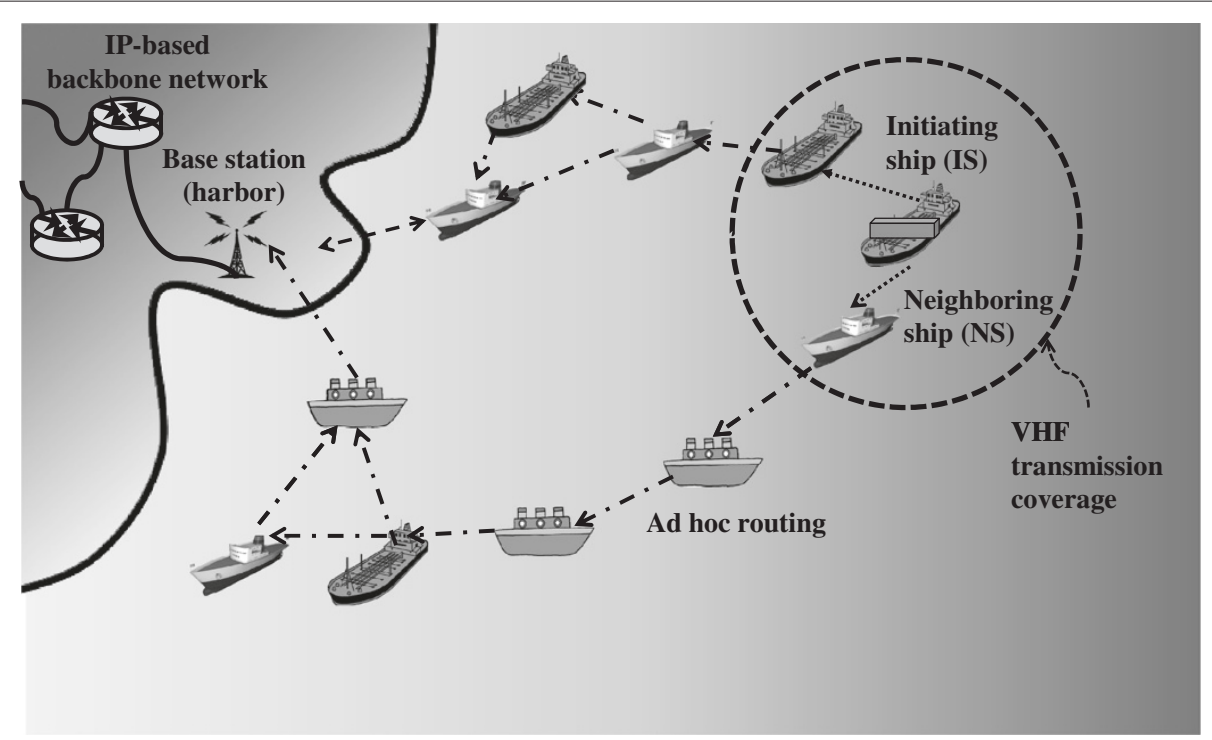

Figure 1 SANET architecture.

along with a specific navigation route. Hence, a SANET is assumed to be an infrastructure-less network in which all ships are randomly but sparsely deployed at sea at any given point in time. Since the transmission distance in VHF communications is tens of kilometers [12], a ship may not directly be connected to a base station (or its destination ship) once the base station is beyond its transmission coverage area. We refer to a ship attempting to connect to a base station as the initiating ship (IS), as shown in Figure 1. If the IS has multiple NSs within its VHF transmission coverage area, these NSs become relay nodes and forward the IS's data to their NSs in an adhoc manner. Finally, the IS's data arrive at the base station. If the IS is unable to locate NSs within its transmission coverage area, it changes its mode of communications to satellite communications in order to achieve seamless multimedia services.

\section{Previous works}

We review SO-TDMA and CS-TDMA in this section. As discussed earlier, these two MAC protocols have been used to provide AIS services. SO-TDMA is mainly used in AIS services (i.e., traffic class A), whereas CS-TDMA is employed to make full use of the frame (i.e., traffic class B) when there is a decrease in traffic class A [13].

\section{SO-TDMA}

Each SO-TDMA frame is divided into 2,250 time slots, and the length of each frame is given as $1 \mathrm{~min}$. The frame is globally fixed, and ships are synchronized to the frame with the help of GPS or Galileo. SO-TDMA is composed of four phases: the initialization phase, the network entry phase (NEP), the first-frame phase, and the continuousoperation phase.

In the initialization phase, a ship listens for channel activity during a frame to find idle time slots. Among idle time slots, nominal transmission slots (NTSs) through which the ship can send its data are determined.

In the NEP, a ship joins the network by determining the initial NTS and sending its data through the first NTS. The following parameters are used to determine the first NTS:

- Nominal start slots (NSSs) refer to randomly selected starting time slots.

- Nominal increment (NIs) refer to periods for selecting available NTSs. NIs are determined by dividing the number of time slots in each frame by the report rate (the desired number of NTSs per frame).

- Selection intervals (SIs) refer to the intervals at which NTSs are determined. SIs are calculated as $0.2 \times \mathrm{NI}$

As illustrated in Figure 2, the ship randomly selects NSSs after the initialization phase and determines the SI around the NSSs. The ship then chooses NTSs among idle time slots within the SI and executes data transfers through the NTSs. If all time slots within the SI are occupied, the time slot assigned by a ship, which is furthest away from the ship, is chosen.

In the first-frame phase, the ship selects the remaining NTSs within NIs. For this, the ship chooses nominal slots (NSs), which correspond to NSSs in the NEP, and are as much as an NI away from the NSS. The ship then selects another NTS among idle time slots in the SI and sends data through the NTS, as shown in Figure 2. The rest of 


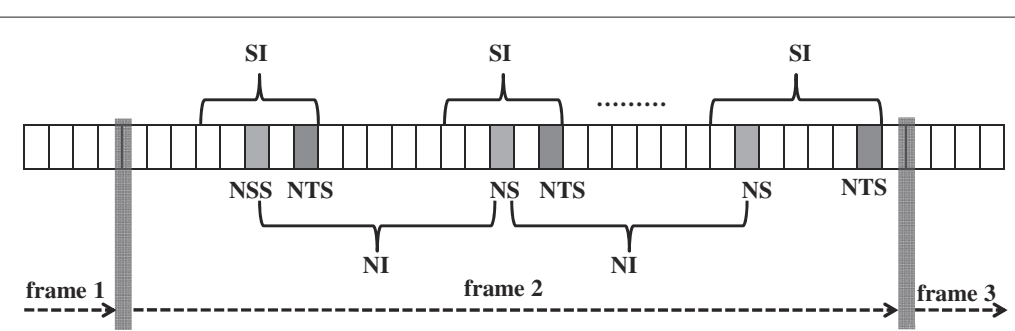

Figure 2 Description of frames in SO-TDMA.

the NTSs are determined by the report rate. For instance, a report rate of 10 (messages per frame) implies that a ship can reserve 10 NTSs for each frame. This repeats until a frame has elapsed and all NTSs are determined. If there is a change in the report rate during any phase, the ship returns to the NEP because the NI depends on the report rate.

In the continuous-operation phase, the ship continuously sends its data through the NTSs previously determined in the network entry and first-frame phases. During the first-frame phase, the ship draws a random integer $n(n=3,4, \ldots, 8)$ for each NTS. After one NTS has been used for $n$ frames in the continuous-operation phase, a new NTS is selected within the same SI as the original NTS. Accordingly, the ship again returns to the network entry and first-frame phases. This is executed in order to avoid receiver collisions between ships by changes in their locations.

\section{CS-TDMA}

The execution of CS-TDMA is similar to SO-TDMA, except for the number of reserved time slots and report intervals. The new parameters for CS-TDMA are as follows.

- Candidate periods (CPs) correspond to NTSs in SO-TDMA.

- Report intervals (RIs) correspond to the report rate in SO-TDMA. When a ship reserves NTSs, which can be equal to the report rate, in SO-TDMA, it requires 10 CPs for each RI in CS-TDMA. Thus, the length of each frame in CS-TDMA is determined by dividing the RI by 1 min (i.e., the frame length for SO-TDMA).
- Nominal transmission times (NTTs) correspond to NSSs in SO-TDMA.

- Transmission intervals (TIs) that correspond to SIs in SO-TDMA are obtained as $\frac{R I}{3}$.

In the initialization phase, a ship mainly searches for idle time slots by checking the CS detection threshold. If the power of the signal exceeds the threshold, the time slot is considered occupied. In the NEP, the ship randomly selects NTTs and determines the TI around the NTT, as illustrated in Figure 3. Within the TI, the ship selects one $\mathrm{CP}$ and sends data through the CP. In the first-frame phase, the ship chooses 10 CPs and sends data through these CPs. The continuous-operation phase of CS-TDMA is similar to that of SO-TDMA.

\section{ASO-TDMA for SANETs}

ASO-TDMA consists of several phases, including the initialization phase (IP), the NEP, and the data transmission phase (DTP). In this section, we describe the frame structures in ASO-TDMA and explain these three phases. Table 1 specifies all the network parameters.

\section{Frame structure}

We define a hop before discussing the frame structures in ASO-TDMA. A hop is defined as a zone determined by the distance from a base station. Assuming that the maximum VHF transmission distance is $30 \mathrm{~km}$, an area that is within $30 \mathrm{~km}$ from a base station belongs to hop 1 . Similarly, an area within $30-60 \mathrm{~km}$ from a base station belongs to hop 2. Thus, the further the area is away from a base station, the larger the number of hop is.

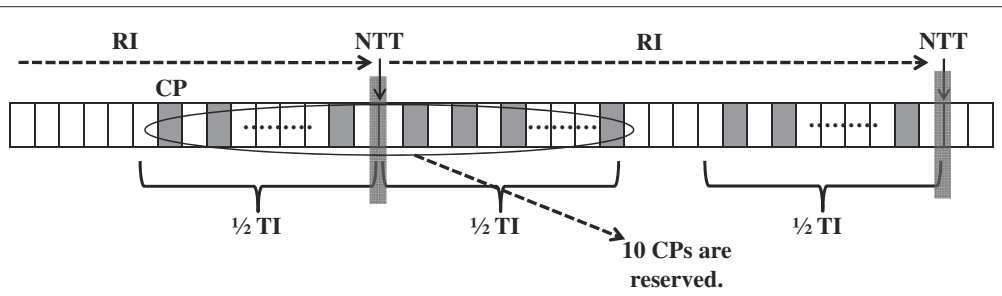

Figure 3 Description of frames in CS-TDMA. 
Table 1 Network parameters

\begin{tabular}{ll}
\hline Parameters & Description \\
\hline$N$ & The number of ships \\
$i$ & The index of ships $(1 \leq i \leq N)$ \\
$m$ & The number of hops (or sub-frames) \\
$h_{i}$ & The hop of ship $i$ \\
$k$ & The index of hops (or sub-frames) $(1 \leq k \leq m)$ \\
$N_{k}$ & The number of time slots belonging to sub-frame $k$ \\
$N_{A f}$ & The number of time slots in one frame for ASO-TDMA \\
\hline
\end{tabular}

Frames in ASO-TDMA differs from those in SO-TDMA and CS-TDMA. Each frame is divided into $m$ sub-frames, which individually correspond to $m$ hops, as shown in Figure 4 . That is, sub-frame $k$ corresponds to hop $k$. This frame fragmentation with respect to hops is intended to reduce the possibility of receiver collisions between ships, and the next section provides a further explanation. As in SO-TDMA and CS-TDMA, each frame in ASO-TDMA consists of multiple time slots such that the number of time slots in each frame is determined as $N_{A f}$, and there are $N_{k}$ time slots in sub-frame $k$. Because ships are generally found near the shore, lower numbered sub-frames receive more time slots: $N_{1}>N_{2}>\cdots>N_{m-1}>N_{m}$ $\left(N_{1}+N_{2}+\cdots+N_{m-1}+N_{m}=N_{A f}\right)$.

The length of the time slot can be determined by considering the maximum propagation delay, the transmission delay (i.e., the data rate and the data packet size), and the switching time specified in [7]. In addition, the values of $m, N_{A f}$, and $N_{k}$ need to be determined by considering network environments such as regional ship density or ship navigation routes. As in SO-TDMA and CS-TDMA, frames in ASO-TDMA are globally fixed, and ships join the network by using their GPS.

\section{Initialization phase}

Every ship currently at sea belongs to a hop, and its hop number is determined by its location. In the initialization phase, ship $i$ determines its hop $h_{i}$ by assuming that all ships have information on the location of a base station. By using its GPS, ship $i$ can obtain its location, and thus calculate the straight-line distance $D_{i}$ between itself and a base station. Then, ship $i$ obtains $h_{i}$ as

$$
h_{i}=\left\lceil\frac{D_{i}}{30 \mathrm{~km}}\right\rceil \text {. }
$$

In addition, ship $i$ scans one frame to locate idle time slots and checks the existence of NSs. If ship $i$ does not detect any NS in the current frame, then it returns to the initialization phase in the next frame. If ship $i$ detects NSs in the current frame, it determines available time slots (ATSs) for the data transfer, which correspond to NTSs in SO-TDMA and CPs in CS-TDMA. ATSs for ship $i$ are determined based on the following rules for assigning time slots.

- If ship $i$ belongs to hop $k$ (i.e., $h_{i}=k$ ), it generally selects available sub-frames whereby it avoids receiver collisions with other ships that concurrently occupy the same time slot as ship $i$ and send their data to the same NSs as ship $i$.

- For a better understanding, we consider the illustration in Figure 5. Ship 1 belongs hop 1, ships 2 and 3 belong to hop 2 , and ship 4 belongs to hop 3 . In addition, ship 2 is an NS of ships 1, 3, and 4. If ships 1 , 3 , and 4 can select their ATS during the same sub-frame, then it is possible for them to choose the same ATS in the sub-frame. Thus, receiver collisions can occur for ship 2 if ships 1, 3, and 4 simultaneously send their data through the same ATS during the same sub-frame. To avoid receiver collisions, the three ships cannot use the same sub-frame at the same time. By contrast, ship 5 does not risk any receiver collision with ship 1 , and thus, ships 5 and 1 can use the same ATS and sub-frame.

- Accordingly, the indices for the available sub-frames of ship $i, S F_{i}$, which avoids receiver collisions, can be expressed as

$$
S F_{i}= \begin{cases}k & \\ k-3 l, & 0<l \leq\left\lfloor\frac{k}{3}\right\rfloor \\ k+3 l, & 0<l \leq\left\lfloor\frac{m-k}{3}\right\rfloor .\end{cases}
$$

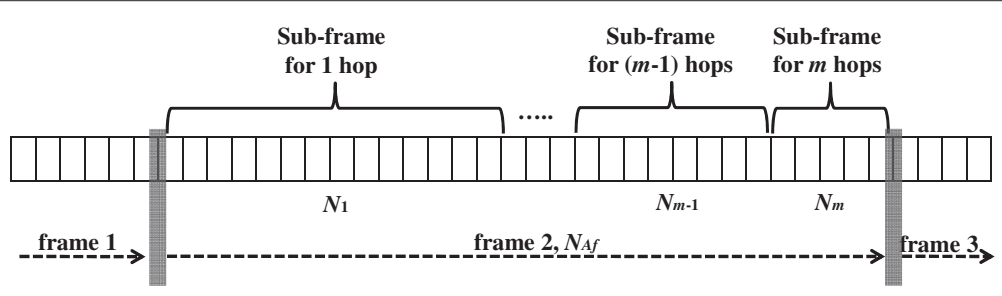

Figure 4 Description of frames in ASO-TDMA. 


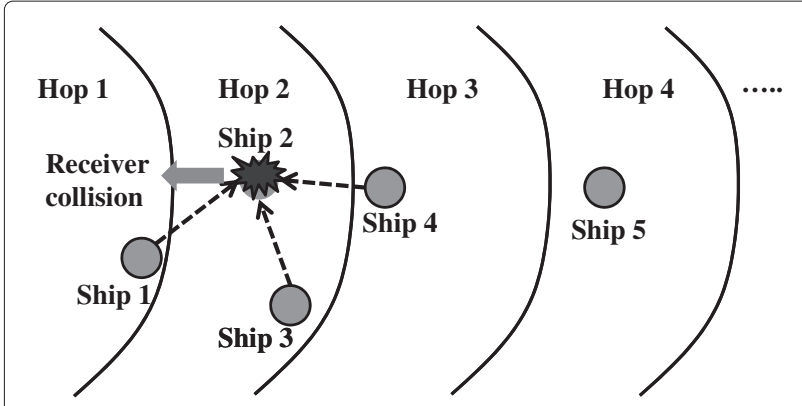

Figure 5 An illustration of a receiver collision.

where $l$ is a positive integer. Equation (2) implies that a ship cannot share sub-frames with ships located 1 or 2 hops away from itself.

- Ship $i$ randomly selects one ATS among idle time slots in each available sub-frame.

- If there are no idle time slots in one available sub-frame, ship $i$ skips to select an ATS in the sub-frame.

- If ship $i$ has $x$ available sub-frames, then it can reserve $x$ ATSs at the maximum.

- For instance, if $m=10$, then a ship belonging to hop 1 has an opportunity to reserve ATSs in sub-frames 1 , 4,7 , and 10 . Thus, at most, the ship can reserve four ATSs.

If ship $i$ cannot find any idle time slots in all available sub-frames in the current frame, it returns to the initialization phase again in the next frame.

\section{NEP}

In the NEP, a ship joins the network by sending any packet through all ATSs determined in the previous frame. Thus, the NEP is executed during the frame immediately following the initialization phase. A ship can send a routing request packet before it sends its data packet. We do not specify any routing approach because we focus on explaining a MAC protocol in this article. A ship can also send its own data packet as an IS and forward the data packet as a relaying ship after determining a routing path. Unlike in SO-TDMA and CS-TDMA, a ship does not have to draw a random number in order to periodically change its occupied time slots because ASO-TDMA attempts to avoid receiver collisions through the proposed rules for assigning time slots. Figure 6 shows a flow chart describing the initialization and network entry phases in ASO-TDMA.

\section{DTP}

The DTP basically repeats the tasks executed in the initialization and network entry phases. A ship continuously determines its hop and available sub-frames, detects NSs, and scans idle time slots to obtain ATSs because of its mobility.

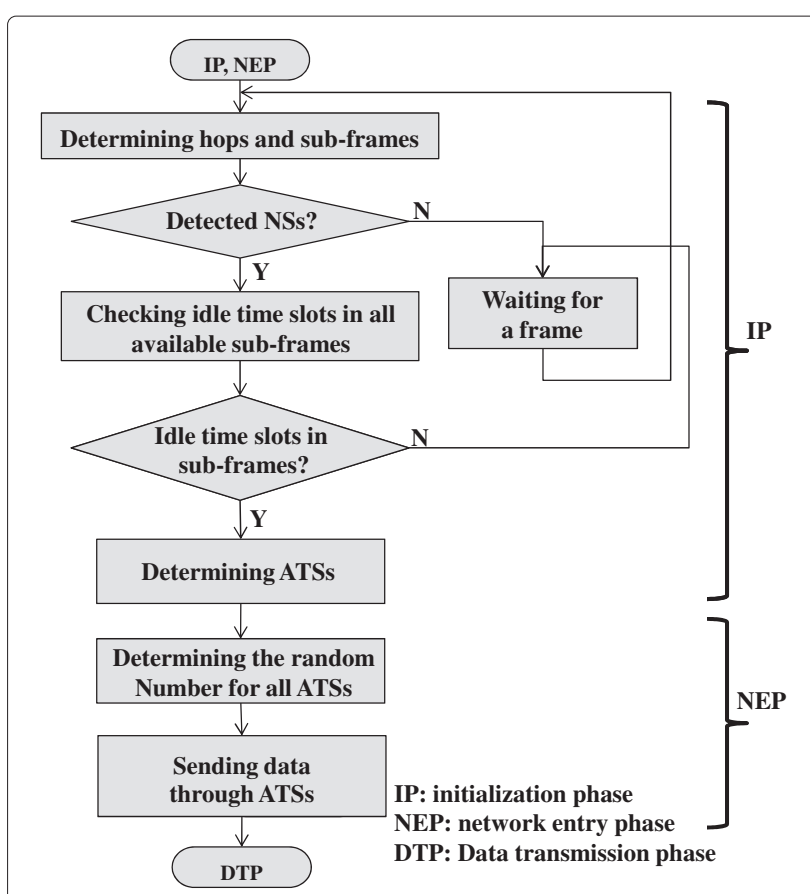

Figure 6 Initialization and network entry phases in ASO-TDMA.

In addition to these tasks, the ship is required to investigate the results of rdt by determining whether its NSs receive information without any corruption. The ship can check the results of rdt via one-hop data or NACK from NSs upon scanning the current frame. The ship's receipt of one-hop data from its NSs during the current frame implies the success of the data transfer in the previous frame. If the ship receives NACK during the current frame, it retransmits the data in the next frame because NACK implies the corruption of transmitted data in the previous frame. If the ship receives neither one-hop data nor NACK after $2 h_{i}$ frames, it retransmits the data. Here, $2 h_{i}$ is heuristically determined such that the waiting time can be adjustable according to the network environment.

If the ship experiences a change, it waits for the next frame and determines new ATSs by scanning the current frame. In addition, if the ship cannot detect any NSs in the current frame, it waits for the next frame by checking the availability of NSs, as illustrated in Figure 7. Finally, if the ship stops the transfer of data transfer, it informs NSs of the release of its ATSs.

\section{Performance analysis}

We analytically investigate the performance of ASOTDMA in terms of the CRs and delays by theoretically deriving two approximate performance parameters under several assumptions. In addition, we compare ASO-TDMA with SO-TDMA and CS-TDMA in terms of the CRs and delays in order to demonstrate the superiority of ASO-TDMA over the two MAC protocols. 


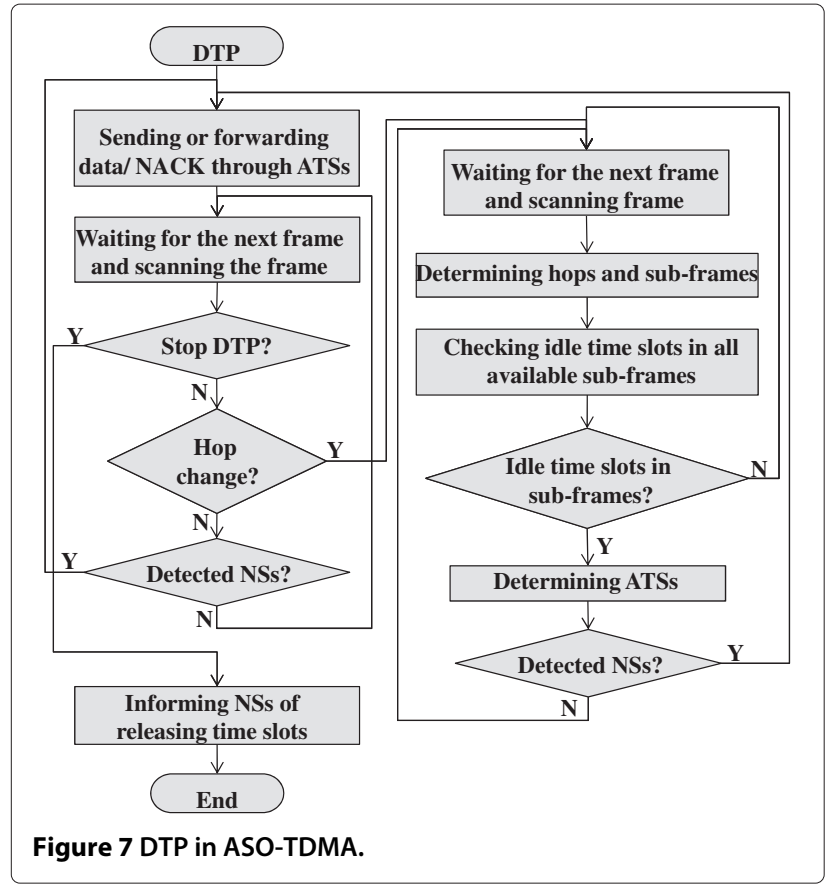

\section{CR}

As discussed earlier, ASO-TDMA can reduce receiver collisions between ships belonging to different hops through the proposed rules for assigning time slots as well as frame fragmentation. However, a ship may experience a receiver collision with another ship belonging to the same hop. That is, ship $i$ in hop $k$ can reserve the same ATS with another ship in hop $k$ that is not an NS of ship $i$, which is referred to as a non-neighboring ship (a nonNS). If these two ships have a common NS and send their data to the NS through the same ATS, then they can experience a receiver collision in the common NS. Because a ship can reserve at most $x$ ATSs in $x$ available sub-frames, it may experience a receiver collision for each ATS in the available sub-frame. To obtain the CR for ASO-TDMA, $C R_{A}$, we define several parameters (Table 2).

We consider ship $i i$ to belong to hop $k$. Ship $i i$ can reserve $x$ ATSs in $x$ sub-frames whose indices are in the set of $X_{k}$. In this case, ship $i i$ can experience a receiver collision with non-NS $j j$ during sub-frame $k k$, which is an element of $X_{k}$. To obtain the CR of ship $i$ for sub-frame

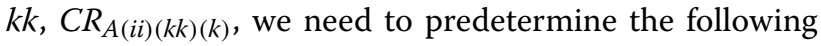
two probabilities:

1. $P_{A 1}$ : The probability that non-NS $j j$ in hop $k$ reserves the same ATS as ship ii during sub-frame $k k$. Non-NS $j j$ can reserve ATSs among ATSs by excluding the time slots occupied by its NSs (as much as $n s_{j j}$ ) in sub-frame $k k$. Assuming that the probability of non-NS $j$ j selecting ATSs among ATSs
Table 2 Parameters for deriving CRs for ASO-TDMA

\begin{tabular}{ll}
\hline Parameters & Description \\
\hline$i i$ & The index of ships located in hop $k$ \\
$n_{k}$ & The number of ships in hop $k$ \\
$n s_{i i}$ & The number of NSs for ship ii \\
$x$ & The number of sub-frames for ship $i i$ \\
$X_{k}$ & The set of sub-frame indices for ship $i i,\left(X_{k}=\right.$ \\
& $\{\ldots, k-3, k, k+3, \ldots\})$ \\
$k k$ & The element of $X_{k},\left(k k \in X_{k}\right)$ \\
$N_{k k}$ & The number of time slots in sub-frame $k k$ \\
$j j$ & $\begin{array}{l}\text { The index of non-NSs of ship ii in hop } k_{,}(1 \leq \\
\left.j j \leq n_{k}-n s_{i i}\right)\end{array}$ \\
$C R_{A(i i)(k k)(k)}$ & $\begin{array}{l}\text { The CR for ASO-TDMA for ship ii in hop } k \text { for } \\
\text { sub-frame } k k\end{array}$ \\
\hline
\end{tabular}

(i.e., $N_{k k}-n s_{j j}$ ) is equiprobable, we can obtain $P_{A 1}$ as

$$
P_{A 1}=\frac{1}{N_{k k}-n s_{j j}} .
$$

2. $P_{A 2}$ : The probability that non-NS $j j$ has the same NS as ship $i$. The number of NSs of Non-NS $j j$ is defined as $n s_{j j}$. One of $n s_{j j}$ NSs can be an NS of ship $i i$. In addition, two of them can be two NSs of ship $i$. Assuming that the number of common NSs between ship ii and non-NS $j j$ is equiprobable, we can express $P_{A 2}$ as

$$
\begin{aligned}
P_{A 2} & =\frac{1}{n s_{j j}} \times\left[1+2+\cdots+\left(n s_{j j}-1\right)+n s_{j j}\right] \\
& =\frac{\left(n s_{j j}+1\right)}{2 n s_{j j}} .
\end{aligned}
$$

For one non-NS $j j$, we can calculate the $\mathrm{CR}$ as $P_{A 1} \times P_{A 2}$. Since there are $n_{k}-n s_{i i}$ non-NSs for ship $i i$, we can express $C R_{A(i i)(k k)(k)}$ as

$$
C R_{A(i i)(k k)(k)}=\sum_{j j=1}^{n_{k}-n s_{i i}}\left[\left(\frac{1}{N_{k k}-n s_{j j}}\right) \times \frac{\left(n s_{j j}+1\right)}{2 n s_{j j}}\right] .
$$

Since ship ii can use $x$ available sub-frames whose indices are elements of $X_{k}$, we can calculate $C R_{A(i i)(k)}$ as

$$
C R_{A(i i)(k)}=\sum_{k k \in X_{k}} C R_{A(i i)(k k)(k)} .
$$

Finally, we can obtain $C R_{A}$ by averaging Equation (6) according to $i i$ and $k$ and express as

$$
C R_{A}=\frac{1}{m}\left\{\sum_{k=1}^{m}\left[\frac{1}{n_{k}} \sum_{i i=1}^{n_{k}} C R_{A(i i)(k)}\right]\right\} .
$$


In order to obtain CRs of SO-TDMA and CS-TDMA $\left(C R_{S}\right.$ and $C R_{C}$, respectively), we first define several parameters (Table 3).

For SO-TDMA, we can obtain $P_{S 1}$ and $P_{S 2}$, which correspond to $P_{A 1}$ and $P_{A 2}$, respectively, as follows.

1. Ship $i$ may experience a receiver collision with a non-NS $j$ that can reserve one NTS by excluding NTSs of its NSs (i.e., $\sum_{q=1}^{n s_{j}} n_{q}$ ) and its remaining NTSs (i.e., $n_{i}-1$ ). Thus, there may be a receiver collision of ship $i$ if non-NS $j$ can reserve one NTS among time slots, whose number can be expressed as $N_{S f}-\sum_{q=1}^{n s_{j}} n_{q}-\left(n_{j}-1\right)$. Assuming that the probability of non-NS $j$ selecting NTS among ATSs is equiprobable, we can calculate $P_{S 1}$ as

$$
P_{S 1}=\frac{1}{N_{S f}-\sum_{q=1}^{n s_{j}} n_{q}-\left(n_{j}-1\right)}
$$

2. As in the case of $P_{A 2}$, we can obtain $P_{S 2}$ by assuming that the number of common NSs between ship $i$ and non-NS $j$ is equiprobable. Thus, we can express $P_{S 2}$ as

$$
P_{S 2}=\frac{\left(n s_{j}+1\right)}{2 n s_{j}} .
$$

Because there are $\left(N-n s_{i}\right)$ non-NSs for ship $i$, we can express the CR of SO-TDMA for ship $i$ for NTS $l, C R_{S(i)(l)}$, as

$$
\begin{aligned}
C R_{S(i)(l)}= & \sum_{j=1}^{N-n s_{i}}\left[\left(\frac{1}{N_{S f}-\sum_{q=1}^{n s_{j}} n_{q}-\left(n_{j}-1\right)}\right)\right. \\
& \left.\times \frac{\left(n s_{j}+1\right)}{2 n s_{j}}\right] .
\end{aligned}
$$

Table 3 Parameters for deriving CRs for SO-TDMA and
CS-TDMA
\begin{tabular}{ll}
\hline Parameters & Description \\
\hline$n_{i}$ & The number of NTSS (CPS) assigned by ship $i$ \\
$l$ & The index of NTSS (CPS) assigned by ship $i,\left(1 \leq l \leq n_{i}\right)$ \\
$n s_{i}$ & The number of NSs for ship $i$ \\
$j$ & The index of non-NSs for ship $i,\left(1 \leq j \leq N-n_{i}\right)$ \\
$n_{j}$ & The number of NTSs assigned by ship $j$ \\
$q$ & The index of NSs for ship $j,\left(1 \leq q \leq n_{j}\right)$ \\
$n_{q}$ & The number of NTSs assigned by ship $q$ \\
$N_{S f}$ & The number of time slots in a frame for SO-TDMA \\
$N_{C f}$ & The CR for SO-TDMA for ship $i$ at NTS / \\
$C R_{S(i)(l)}$ & The CR for CS-TDMA for ship $i$ at CP / \\
$C R_{S(i)(l)}$ &
\end{tabular}

We can determine the CR of SO-TDMA for $i, C R_{S(i)}$, by summing up all $C R_{S(i)(l)} \mathrm{s}$ as

$$
C R_{S(i)}=\sum_{l=1}^{n_{i}} C R_{S(i)(l)} .
$$

Finally, we obtain $C R_{S}$ by averaging Equation (11) with respect to $i$ as

$$
C R_{S}=\frac{1}{N} \sum_{i=1}^{N} C R_{S(i)} .
$$

For CS-TDMA, we can derive $P_{C 1}$ and $P_{C 2}$, which correspond to $P_{A 1}$ and $P_{A 2}$, respectively, as follows.

1. In CS-TDMA, the number of CPs for ship $i, n_{i}$ is fixed to 10 [2]. There may be a receiver collision for ship $i$ if non-NS $j$ can reserve one CP among time slots, whose number can be expressed as $N_{C f}-\sum_{q=1}^{10} n_{q}-(10-1)$. Under the same assumption, we can obtain $P_{C 1}$ as follows.

$$
P_{C 1}=\frac{1}{N_{C f}-10 \times n s_{j}-(10-1)} .
$$

2. As in the case of $P_{S 2}$, we can obtain $P_{C 2}$ as follows.

$$
P_{C 2}=\frac{\left(n s_{j}+1\right)}{2 n s_{j}} \text {. }
$$

Thus, we can derive $C R_{C(i)(l)}$ by using $P_{C 1}$ and $P_{C 2}$. As in the case of SO-TDMA, we can obtain $C R_{C}$ as follows.

$$
\begin{aligned}
C R_{C}= & \frac{1}{N} \sum_{i=1}^{N} \sum_{l=1}^{n_{i}}\left[\frac{1}{N_{C f}-10 \times n s_{j}-(10-1)}\right. \\
& \left.\times \frac{\left(n s_{j}+1\right)}{2 n s_{j}}\right] .
\end{aligned}
$$

\section{Delay}

We now derive delays for ASO-TDMA, SO-TDMA, and CS-TDMA ( $D_{A}, D_{S}$, and $D_{C}$, respectively). Here, we define a delay as the sum of the amount of time a ship spends reserving its ATSs (NTSs or CPs) and the amount of time it spends sending data through the ATSs (NTSs or CPs). Table 4 shows the parameters for deriving $D_{A}, D_{S}$, and $D_{C}$.

In ASO-TDMA, a ship can reserve ATSs if it can locate an NS. That is, the ship has to wait until there is an NS. In addition, when there is a hop change, the ship has to wait one frame to go back to the initialization and network entry phases. Thus, $D_{A}$ depends on whether there is a hop change/an NS.

We assume that a ship successfully completes a data transfer in the previous frame and counts the number of frames $(w)$ after the previous frame $(w \geq 1)$, as illustrated in Figure 8. If the ship has no hop change and detects an NS in the first frame after the data transfer in the previous frame (i.e., frame 1), it can send data during frame 1. 
Table 4 Parameters for deriving delays for ASO-TDMA, SO-TDMA, and CS-TDMA

\begin{tabular}{|c|c|}
\hline Parameters & Description \\
\hline$w$ & The index of frames \\
\hline$P_{N S(A)(W)}$ & $\begin{array}{l}\text { The probability of the ship having its NSs } \\
\text { during frame } k \text { for ASO-TDMA }\end{array}$ \\
\hline$P_{N S(S)(w)}$ & $\begin{array}{l}\text { The probability of the ship having its NSs } \\
\text { during frame } k \text { for SO-TDMA }\end{array}$ \\
\hline$P_{\mathrm{NS}(C)(W)}$ & $\begin{array}{l}\text { The probability of the ship having its NSs } \\
\text { during frame } k \text { for CS-TDMA }\end{array}$ \\
\hline$P_{H(A)(W)}$ & $\begin{array}{l}\text { The probability of the ship experiencing a } \\
\text { hop change during frame } k \text { for ASO-TDMA }\end{array}$ \\
\hline $\mathrm{TS}_{\mathrm{pL}}$ & $\begin{array}{l}\text { The time slot index of the last ATS (or NTS) } \\
\text { in the previous frame for the three MAC } \\
\text { protocols }\end{array}$ \\
\hline $\mathrm{TS}_{\mathrm{WF}}$ & $\begin{array}{l}\text { The time slot index of the first ATS (or NTS) in } \\
\text { frame } w \text { for the three MAC protocols }\end{array}$ \\
\hline $\mathrm{TS}_{\mathrm{wL}}$ & $\begin{array}{l}\text { The time slot index of the last ATS (or NTS) in } \\
\text { frame } w \text { for the three MAC protocols }\end{array}$ \\
\hline$\tau$ & $\begin{array}{l}\text { The length of the time slot for the three MAC } \\
\text { protocols }\end{array}$ \\
\hline
\end{tabular}

Thus, we can obtain $D_{A}$ for frame $1, D_{A 1}$, as follows:

$$
D_{A 1}=\left(1-P_{H(A)(1)}\right) \times P_{N S(A)(1)} \times\left(d_{A 11}+d_{A 12}\right)
$$

where $d_{A 11}$ is the amount of time a ship spends waiting for the first ATS in frame 1 and $d_{A 12}$ is the amount of time a ship spends waiting to use all ATSs in frame 1 . As shown in Figure 8 , we can derive $d_{A 11}$ and $d_{A 12}$ by using the time slot index of corresponding ATSs as follows:

$$
\begin{aligned}
& d_{A 11}=\left(N_{A f}-1+\mathrm{TS}_{1 \mathrm{~F}}-T S_{p L}\right) \times \tau \\
& d_{A 12}=\left[\mathrm{TS}_{1 \mathrm{~L}}-T S_{1 F}+(1-x)\right] \times \tau
\end{aligned}
$$

where $x$ is the number of ATSs. If the ship has a hop change or does not detect any NS until frame 2, then we can derive $D_{A}$ for frame $2, D_{A 2}$, as follows.

$$
\begin{aligned}
D_{A 2}= & \left(1-P_{N S(A)(1)}+P_{H(A)(1)} \times P_{N S(A)(1)}\right) \\
& \times\left[\left(1-P_{H(A)(2)}\right) \times P_{N S(A)(2)}\right] \\
& \times\left(d_{A 21}+d_{A 22}\right)
\end{aligned}
$$

where $d_{A 21}$ is the amount of time a ship spends waiting for the first ATS in frame 2 and $d_{A 22}$ is the amount of time a ship spends waiting while using all ATSs in frame 2. We can express $d_{A 21}$ and $d_{A 22}$ as follows.

$$
\begin{aligned}
& d_{A 21}=\left(2 N_{A f}-1+\mathrm{TS}_{2 \mathrm{~F}}-T S_{p L}\right) \times \tau \\
& d_{A 22}=\left[\mathrm{TS}_{2 \mathrm{~L}}-T S_{2 F}+(1-x)\right] \times \tau .
\end{aligned}
$$

Using Equations (16) and (19), we can generalize $D_{A}$ for frame $w, D_{A w}$, as

$$
\begin{aligned}
D_{A w}= & {\left[\prod_{a=1}^{w-1}\left(1-P_{N S(A)(a)}+P_{H(A)(a)} \times P_{N S(A)(a)}\right)\right] } \\
& \times\left[\left(1-P_{H(A)(w)}\right) \times P_{N S(A)(w)}\right] \\
& \times\left(d_{A w 1}+d_{A w 2}\right) .
\end{aligned}
$$

In addition, $d_{A w 1}$ and $d_{A w 2}$ are also generalized as

$$
\begin{aligned}
& d_{A w 1}=\left(w \times N_{A f}-1+\mathrm{TS}_{\mathrm{wF}}-T S_{p L}\right) \times \tau \\
& d_{A w 2}=\left[\mathrm{TS}_{\mathrm{wL}}-T S_{w F}+(1-x)\right] \times \tau .
\end{aligned}
$$

Finally, we can express $D_{A}$ as

$$
D_{A}=\sum_{w=1}^{\infty} D_{A w} .
$$

In SO-TDMA, a ship has to wait for reserving NTSs until it finds an NS, and thus, $D_{S}$ depends on whether there is an NS. As shown in Figure 9, if a ship detects NSs during the first frame after successfully completing a data transfer in the previous frame, we can obtain $D_{S}$ for frame $1, D_{S 1}$, as follows:

$$
D_{S 1}=P_{N S(S)(1)} \times\left(d_{S 11}+d_{S 12}\right)
$$

where $d_{S 11}$ is the amount of time a ship spends waiting for the first NTS in frame 1 and $d_{S 12}$ is the amount of time a ship spends waiting while using all NTSs in frame 1 . As in the cases of $d_{A 11}$ and $d_{A 12}$, we can express $d_{S 11}$ and $d_{S 12}$ as

$$
\begin{aligned}
& d_{S 11}=\left(N_{S f}-1+\mathrm{TS}_{1 \mathrm{~F}}-T S_{p L}\right) \times \tau \\
& d_{S 12}=\left[\mathrm{TS}_{1 \mathrm{~L}}-T S_{1 F}+\left(1-n_{S}\right)\right] \times \tau
\end{aligned}
$$

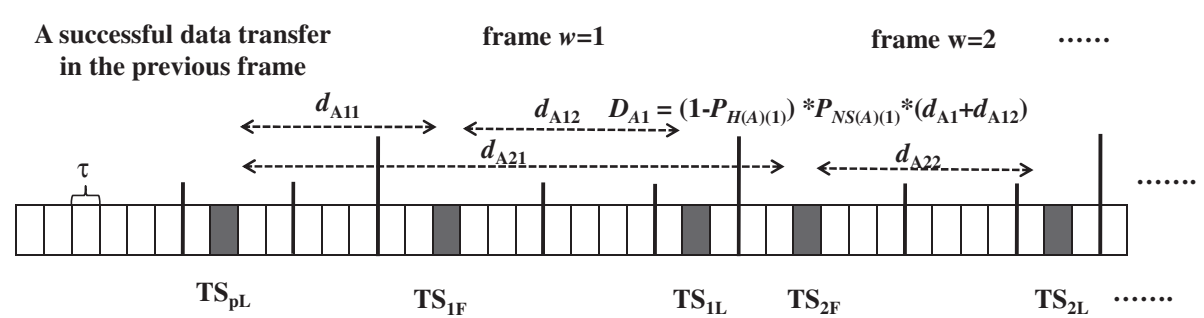

Figure 8 Description of frames for obtaining delays for ASO-TDMA. 


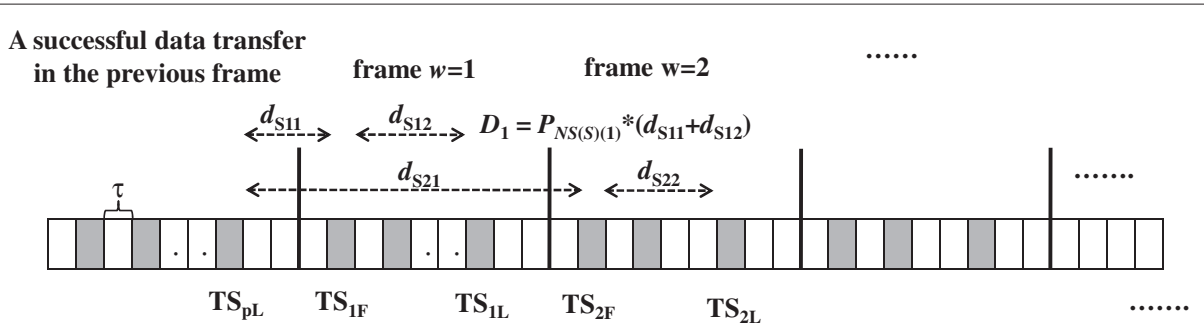

Figure 9 Description of frames for obtaining delays for SO-TDMA.

where $n_{s}$ is the number of NTSs for a ship. As in the case of $D_{A w}$, we can generalize $D_{S}$ for frame $w, D_{S w}$, as

$$
\begin{aligned}
D_{S w}= & {\left[\prod_{a=1}^{w-1}\left(1-P_{N S(S)(a)}\right)\right] } \\
& \times P_{N S(S)(w)} \times\left(d_{S w 1}+d_{S w 2}\right)
\end{aligned}
$$

where $d_{S w 1}$ and $d_{S w 2}$ are calculated as

$$
\begin{aligned}
& d_{S w 1}=\left(w \times N_{S f}-1+\mathrm{TS}_{\mathrm{wF}}-T S_{p L}\right) \times \tau \\
& d_{S w 2}=\left[\mathrm{TS}_{\mathrm{wL}}-T S_{w F}+\left(1-n_{s}\right)\right] \times \tau .
\end{aligned}
$$

Finally, we can express $D_{S}$ as

$$
D_{S}=\sum_{w=1}^{\infty} D_{S w}
$$

In CS-TDMA, $D_{C}$ (like $D_{S}$ ) depends on whether there is an NS. As shown in Figure 10, we determine $D_{C}$ based on RIs, not frames, because CPs are chosen in the RI cycle. We define the index of RI as $v$ and draw the relationship between $w$ and $v$ to be $w=\lfloor y \times v\rfloor$, where $y=\frac{R I}{1 \min }$ [2]. Accordingly, $N_{C f}$ is given as $\lfloor y\rfloor \times N_{S f}$.

As shown in Figure 10, if a ship detects an NS during the first RI after the success of the previous RI, we can obtain $D_{C}$ for RI $1, D_{C 1}$, as follows.

$$
D_{C 1}=P_{N S(C)(1)} \times\left(d_{C 11}+d_{C 12}\right)
$$

where

$$
\begin{aligned}
& d_{C v 1}=\left(\lfloor y \times 1\rfloor \times N_{S f}-1+\mathrm{TS}_{\mathrm{wF}}-\mathrm{TS}_{\mathrm{pL}}\right) \times \tau( \\
& d_{C 12}=\left[\mathrm{TS}_{1 \mathrm{~L}}-T S_{1 F}+(1-10)\right] \times \tau
\end{aligned}
$$

As in the case of $D_{S w}$, we can generalize $D_{C}$ for RI $v, D_{C v}$, as

$$
\begin{aligned}
D_{C v}= & {\left[\prod_{a=1}^{v-1}\left(1-P_{N S(C)(a)}\right)\right] \times P_{N S(S)(v)} } \\
& \times\left(d_{C v 1}+d_{C v 2}\right)
\end{aligned}
$$

where

$$
\begin{aligned}
& d_{C v 1}=\left(\lfloor y \times v\rfloor \times N_{S f}-1+\mathrm{TS}_{w F}-\mathrm{TS}_{p L}\right) \times \tau \\
& d_{C v 2}=\left[\mathrm{TS}_{\mathrm{wL}}-T S_{w F}+(1-10)\right] \times \tau .
\end{aligned}
$$

Finally, we can derive $D_{C}$ as follows.

$$
D_{C}=\sum_{v=1}^{\infty} D_{C v}
$$

\section{Performance evaluation}

We compare the three MAC protocols (i.e., ASO-TDMA, SO-TDMA, and CS-TDMA) in terms of CRs and delays by using the derived performance parameters. For performance evaluation, we consider the following conditions.

\section{Conditions}

As shown in Figure 11, we model the following five types of ships to investigate the CRs and delays of the three MAC protocols. For this, we assume that any location of a ship can be expressed as two coordinates (X, Y). Since we consider $m$ hops, we assume $X_{\max }$ and $Y_{\max }$ to be $m \times 30 \mathrm{~km}$ and $\mathrm{X}_{0}$ and $\mathrm{Y}_{0}$ to be 0 .

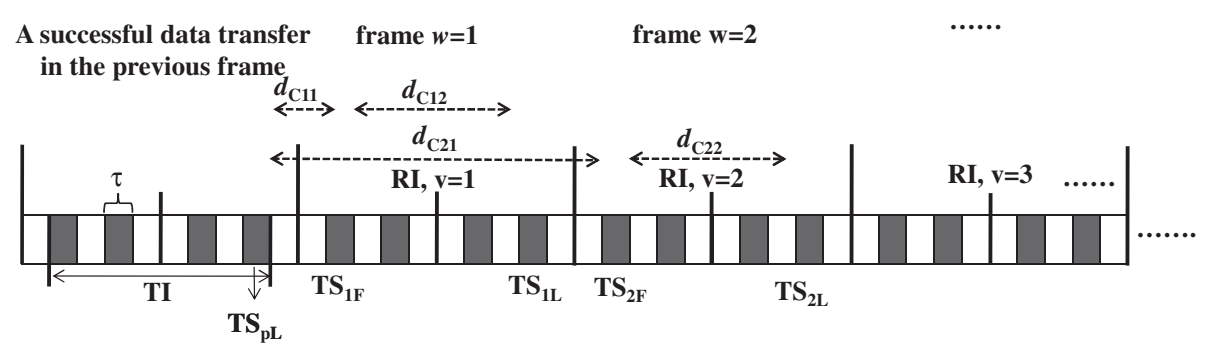

Figure 10 Description of frames for obtaining delays for CS-TDMA. 


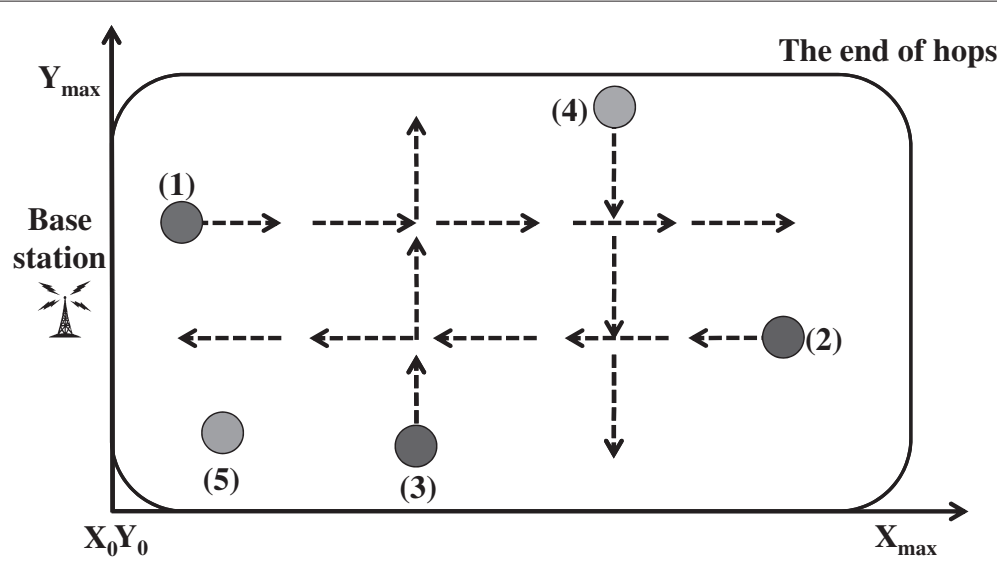

Figure 11 Ship types for performance evaluation.

- Ship type (1): Ships moving toward the sea with the initial position $\left(X_{0}, Y_{R}\right)$, where $Y_{R}$ is a random number uniformly distributed between $Y_{0}$ and $Y_{\max }$.

- Ship type (2): Ships moving toward the shore with the initial position $\left(X_{\max }, Y_{R}\right)$.

- Ship type (3): Ships moving upstream along the shore with the initial position $\left(X_{R}, Y_{0}\right)$, where $X_{R}$ is a random number uniformly distributed between $X_{0}$ and $X_{\max }$.

- Ship type (4): Ships moving downstream along the shore with the initial position of $\left(X_{R}, Y_{\max }\right)$.

- Ship type (5): Ships moving randomly in any direction with the initial position of $\left(X_{R}, Y_{R}\right)$.

With these five types of ships, we consider the following conditions.

- $N_{(1)}, N_{(2)}, N_{(3)}, N_{(4)}$, and $N_{(5)}$ indicate the numbers of ships belonging to the five types of ships, respectively, where $N_{(1)}+N_{(2)}+N_{(3)}+N_{(4)}+N_{(5)}=N$.

- $N_{(1)}, N_{(2)}, N_{(3)}, N_{(4)}$, and $N_{(5)}$ are determined to satisfy with $N_{(1)}=N_{(2)}=N_{(3)}=N_{(4)}<N_{(5)}$.

- $N_{(5)}$ is also given as $N_{1(5)}+N_{2(5)}+\cdots+N_{m(5)}$, where $N_{m(5)}$ corresponds to the number of Type 5 ships belonging to hop $m$. Because ships tend to be located near base stations, we assume that $N_{1(5)}>N_{2(5)}>\cdots>N_{m(5)}$.

- When any ship type reaches a border (e.g., $X_{\max }$ for Type 1 ships), the ship type is dropped, and a new ship with the same ship type is added. Thus, $N$ still holds.

- For all ship types, the velocity is assumed to be a random number with the same uniform distribution

Based on the above model of ship types, we consider the following conditions for numerical analysis.

- $m$ is given as 5 .

- The velocity of each ship is uniformly distributed from 10 to 30 knots.
- The RI for CS-TDMA is given as one minute, that is, the length of each frame in CS-TDMA is the same as that in SO-TDMA.

- $N_{S f}$ and $\tau$ are given as 2,250 and $\frac{60}{2250}$ s, respectively, as defined in [2].

- For any $N, N_{(1)}=N_{(2)}=N_{(3)}=N_{(4)}=0.1 \times N_{(5)}$. In addition, for any $N_{(5)}, N_{1(5)}>N_{2(5)}>\cdots>N_{m(5)}$, and $N_{1(5)}+N_{2(5)}+N_{3(5)}$ is $0.8 \times N_{(5)}$.

- For any $N_{A f}$ in ASO-TDMA, $N_{1}>N_{2}>\cdots>N_{m}$, and $N_{1}+N_{2}+N_{3}$ is $0.8 \times N$.

\section{CR}

Figure 12 shows the CRs for the three MAC protocols with respect to the number of ships. An increase in $N$ increases the CR for all three MAC protocols. When $N_{A f}$ and $N_{S f}$ are the same (i.e., 2,250), ASO-TDMA reduces the CR by as much as $30 \%$ in comparison with SO-TDMA and

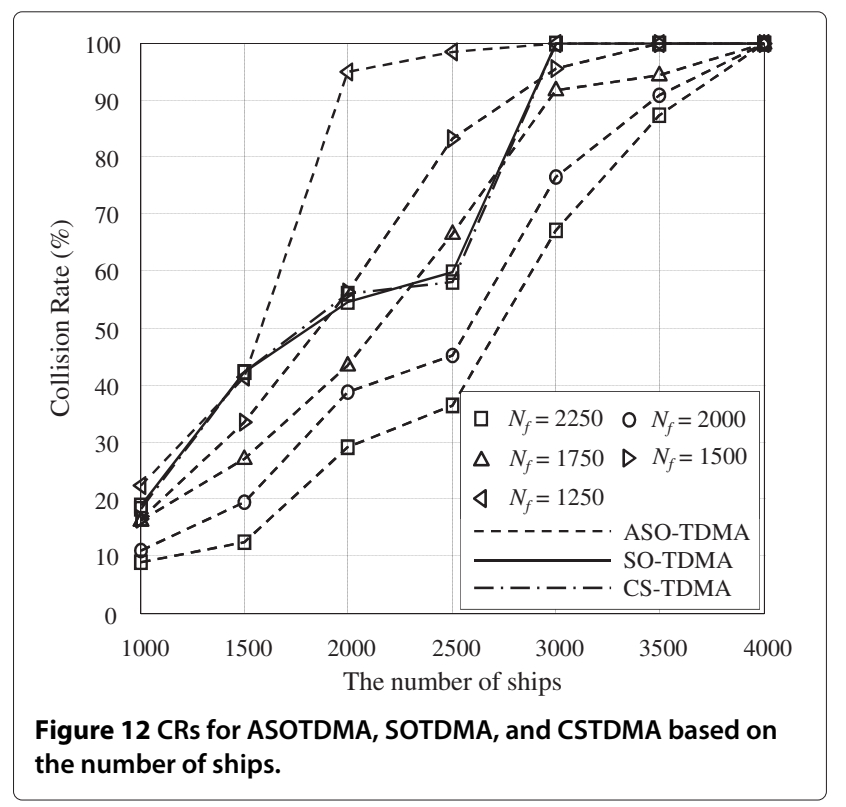




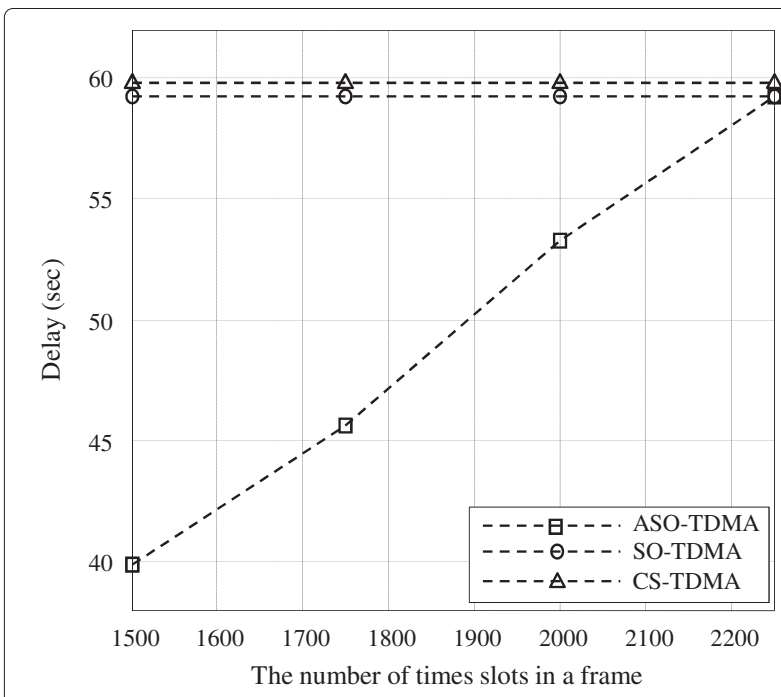

Figure 13 Delays for ASOTDMA, SOTDMA, and CSTDMA based on the number of time slots in each frame.

CS-TDMA. This result indicates that ASO-TDMA based on the proposed rules for assigning time slots and frame fragmentation is more likely to reduce collisions between ships than SO-TDMA and CS-TDMA.

We determine whether we can further reduce the number of time slots in each frame, which can directly influence delay performance. That is, the smaller the number of time slots in a frame, the shorter the delay is. As shown in Figure 12, the three MAC protocols show similar CRs when $N_{A f}$ is 1,750 . If $N_{A f}$ is 1,750 for ASO-TDMA, then $D_{A}$ can be lower than $D_{S}$ or $D_{C}$. If $N_{A f}$ exceeds 1,750 for ASO-TDMA, then $C_{A}$ can be lower than $C_{S}$ or $C_{C}$. Thus, there is a performance trade-off between CRs and delays based on changes in $N_{A f}$. We now examine how delay performance varies according to $N_{A f}$ to clarify this performance trade-off.

\section{Delay}

Figure 13 illustrates the delays of the three MAC protocols according to the number of time slots in each frame. A decrease in $N_{A f}$ reduces delays because the amount of time a ship spends waiting while reserving ATSs decreases. As discussed earlier, an $N_{A f}$ value of 1,750 ensures that the CR for ASO-TDMA is similar to those for SO-TDMA and CS-TDMA. In this case, $D_{A}$ is approximately $26 \%$ lower than $D_{S}$ and $D_{C}$. Although $N_{A f}$ is identical to $N_{S f}, D_{A}$ is still slightly lower than $D_{S}$ and $D_{C}$. This implies that ASO-TDMA can provide SANETs with superior performance in terms of delays and $C R$ performances compared to SO-TDMA and CS-TDMA.

\section{Conclusions}

This article proposed ASO-TDMA, a MAC protocol targeting SANETs. ASO-TDMA supports fragmented frame structures consisting of several sub-frames according to hops. In addition, ASO-TDMA provides ships with important rules for assigning time slots that can reduce receiver collisions, which is a serious problem in SOTDMA and CS-TDMA. The article derives some performance parameters to compare the three MAC protocols in terms of CRs and delays and five types of models regarding ships in a SANET for numerical analysis. The results indicate that, all else being equal, ASO-TDMA outperforms SO-TDMA and CS-TDMA in both CRs and delays. In addition, there is a performance trade-off between CRs and delays according to the number of time slots in each frame, implying that, give the same delay (CR), the CR (delay) is lower (shorter) for ASO-TDMA than for SO-TDMA and CS-TDMA.

Thus, ASO-TDMA should be an effective MAC protocol for SANETs, which are designed to provide ships with diverse maritime multimedia services. In addition, the results of the numerical analysis for CRs and delays can be used as an engineering table for designing SANETs based on ASO-TDMA. Finally, this article's performance analysis, which considers several types of ships, can be applied to any MAC protocol for SANETs and extended to other network settings.

\section{Competing interests}

The authors declare that they have no competing interests.

\section{Acknowledgements}

This study was conducted as a part of the research projects of "Development of marine RF-based ad-hoc network for ship(1615001812)" financially supported by the Ministry of Land, Transport and Maritime Affairs (MLTM) of Korea.

Received: 17 January 2012 Accepted: 31 August 2012

Published: 25 October 2012

\section{References}

1. IMO IC970E, global maritime distress and safety system (GMDSS) Manual. 4 th edn, (2009)

2. Rec. ITU-R M.1371-4, Technical characteristics for a universal ship borne automatic identification system using time division multiple access in the VHF maritime mobile band (2010)

3. A Sarolic, A review of maritime navigation and radiocommunication equipment and systems standardization. in IEEE Int. Symposium Electronics in Marine, ELMAR 2004, (June 2004) pp. 380-383

4. IEC 61097-6, global maritime distress and safety system (GMDSS)-Part 6 : Narrowband direct-printing telegraph equipment for the reception of navigational and meteorological warnings and urgent information to ships (NAVTEX). edn. 2.1, (2012)

5. C Yun, A Cho, S Kim, J Park, Y Lim, Design of multiband maritime network for ships and its applications. Int. J. KIMICS. 7(3), 314-322 (2009)

6. RESOLUTION 951 (Rev.WRC-07), Enhancing the international spectrum regulatory framework (2007)

7. Rec. ITU-R M. 1842-1, Characteristics of VHF radio systems and equipment for the exchange of data and electronic mail in the maritime mobile service RR Appendix 18 channels (2009)

8. Rec. ITU-R M. 489-2, Technical characteristics of VHF radiotelephone equipment operating in the maritime mobile service in channels spaced by $25 \mathrm{kHz}(1995)$

9. IEEE 802.11a, Supplement to IEEE Standard for Information technology-telecommunications and information exchange between systems-local and metropolitan area networks-Specific requirements- 
Part11: Wireless LAN Medium access control (MAC) and Physical Layer

(PHY) Specifications: High-speed Physical layer in the $5 \mathrm{GHz}$ band (1999)

10. JF Kurose, KW Ross, Computer Networking. 3rd edn. (New York, US, PEARSON, Addison Wesley, 2005)

11. WM Moh, D Yao, K Makki, Analyzing the hidden-terminal effects and multimedia support for wireless LAN. Comput. Commun. 23(10), 998-1013 (2000)

12. http://en.wikipedia.org/wiki/VHF

13. http://en.wikipedia.org/wiki/AutomaticldentificationSystem

doi:10.1186/1687-1499-2012-320

Cite this article as: Yun and Lim: ASO-TDMA: ad-hoc self-organizing TDMA protocol for shipborne ad-hoc networks. EURASIP Journal on Wireless Communications and Networking 2012 2012:320.

\section{Submit your manuscript to a SpringerOpen ${ }^{\circ}$ journal and benefit from:}

- Convenient online submission

Rigorous peer review

- Immediate publication on acceptance

- Open access: articles freely available online

- High visibility within the field

- Retaining the copyright to your article 